\title{
Injertos en rinoseptoplastía: Experiencia del Departamento de Otorrinolaringología de la Pontificia Universidad Católica de Chile
}

\author{
Grafts in rinoplasty: \\ Pontificia Universidad Católica de Chile. ENT Department Experience
}

Gabriel Faba $\mathbf{C}^{1}$, Santiago Cornejo Sch ${ }^{1}$, Daniela Gutiérrez $\mathbf{C}^{1}$, Hayo Breinbauer $\mathbf{K}^{\mathbf{1}}$.

\begin{abstract}
RESUMEN
Introducción: La rinoplastía es una de las cirugías estéticas más desafiantes. Dentro de las alternativas quirúrgicas destaca el uso de diversos injertos e implantes los cuales debiesen ser conocidos por todos los cirujanos que realizan esta cirugía.

Objetivo: Describir la experiencia del Departamento de Otorrinolaringología de la Pontificia Universidad Católica de Chile en el uso de injertos en rinoplastías.

Material y método: Estudio retrospectivo descriptivo donde se revisaron protocolos operatorios de todos los pacientes operados en dicho comité entre marzo 2008 y agosto 2009.

Resultados: Se realizaron 98 rinoseptoplastías por rinoseptodesviación en el período, siendo el $84 \%$ por abordaje abierto. El injerto más utilizado fue el vástago de columela en el $84 \%$ de todas las rinoplastías, seguido por el escudo anterior a la punta nasal $(28 \%)$ y el espaciador entre laterales superiores y tabique (18\%). Injertos alares, de dorso, de tabique y otros de refinamiento de punta fueron utilizados en menos del $5 \%$ de los casos. En todos los casos se utilizó injertos autólogos (tabique nasal). La gran mayoría de los injertos fue utilizada por abordaje abierto. Al momento de la revisión no hubo complicaciones que se puedan atribuir específicamente al uso de injertos.
\end{abstract}

Discusión: Nuestra experiencia sigue la tendencia de "resecar menos" y rellenar. El uso de injertos resulta valioso dentro del repertorio quirúrgico en rinoplastía.

Palabras clave: Rinoplastía, rinoseptoplastía, injertos.

\begin{abstract}
Introduction: Rhinoplasty is one of the most challenging cosmetic surgeries. Among surgical alternatives the vast variety of graft and implants are of great value, and every surgeon should have a thorough knowledge of the most commonly used.

Aim: To describe the experience in the use of grafts in rhinoplasty by the Pontificia Universidad Catolica de Chile "Nose Committee", ENT Department.
\end{abstract}

${ }^{1}$ Médico. Departamento Otorrinolaringología, Pontificia Universidad Católica de Chile. 
Material and method: Retrospective study. All committee patient's surgical protocols between March 2008 and August 2009 were reviewed.

Results: 98 rhinoseptoplasties due to rhinoseptedeviation were conducted during the analized period. $84 \%$ were conducted through an open approach. Columella strut was the most commonly used graft (84\%), followed by the shield graft (28\%) and the spreader (18\%). Alar, nasion and septal correcting were used in less than $5 \%$. Autologous grafts (nasal septum) were used in all grafts. The vast majority of the grafts were implemented through an open approach. At the time of this review no graft related complications were reported.

Discussion: Our "Nose Committee's experience follows the trend of "cut less" and fill. Grafts represent a valuable asset in a rhinoplasty surgeon repertoire.

Key words: Rhinoplasty, Rhinoseptoplasty, grafts.

\section{INTRODUCCIÓN}

La rinoseptoplastía es reconocida como uno de los procedimientos más difíciles dentro de la cirugía plástica facial. Su objetivo último es lograr una nariz de apariencia natural y estéticamente agradable, manteniendo a su vez una vía aérea funcional ${ }^{1-2}$.

Para alcanzar este propósito, el uso de implantes e injertos puede ser de enorme utilidad dentro del repertorio de un cirujano en una rinoplastía, debiendo éste profundizar su conocimiento sobre los materiales y técnicas comúnmente utilizados en la actualidad ${ }^{2-3}$.

A modo de concepto, un injerto ideal debiese reunir una serie de características (Tabla 1) que ningún material en la actualidad es capaz de satisfacer.
Los distintos materiales disponibles pueden ser clasificados en 3 fuentes principales: orgánicos, sintéticos y productos de ingeniería biomédica de tejidos ${ }^{1}$.

Injertos orgánicos: Los que a su vez pueden ser subclasificados en:

1. Autoinjertos: Se obtienen del mismo individuo 0 de una persona genéticamente idéntica. Son el tipo de injertos de mayor biointegración y ausencia de reacciones adversas ${ }^{4}$.Dependiendo del sitio donante presentan diferentes grados de rigidez, flexibilidad y duración. Destacan el cartílago autólogo (principalmente de cartílago septal, auricular 0 de parrilla costal. Estos siguen siendo para la mayoría de los autores el mejor material de injerto en la actualidad ${ }^{5}$ ), los

Tabla 1. Características de un injerto ideal ${ }^{1}$

\begin{tabular}{|ll|}
\hline Características macroscópicas & Características microscópicas \\
\hline - No crea ningún tipo de morbilidad en el sitio del injerto & - Debe ser fácilmente biointegrado por el huésped \\
- Fácilmente disponible & - Ser inerte, puro y carente de toda contaminación que pueda \\
- Barato & promover una reacción inflamatoria \\
- Fácil de tallar & No ser carcinogénico ni tampoco catalizador de enfermedades \\
- Se fija a la zona receptora sin dificultad & - No debe ser un vector que transmita agentes infecciosos \\
- Mantiene su forma en el tiempo sin perder su volumen o & como la hepatitis o VIH \\
alterar sus propiedades físicas & Debe ser resistente a la infección \\
Tiene un balance adecuado entre rigidez y flexibilidad & subproductos peligrosos. \\
\hline
\end{tabular}


injertos de hueso autógeno craneal y los injertos autólogos de fascia.

2. Aloinjertos u homoinjertos: Obtenidos de individuo 0 de una persona genéticamente diferente, pero pertenecientes a la misma especie. Dentro de ellos destacan los injertos de cartílago costal irradiados (a partir de donantes cadavéricos, pero con una aparente alta tasa de reabsorción ${ }^{6}$ ) y la dermis acelular humana ("Alloderm", también de origen cadavérico, con utilidad en perforaciones septales y bordes osteocartilaginosos muy marcados)

3. Xenoinjertos: Se obtienen a partir de organismos pertenecientes a una especie diferente. Se utilizan muy rara vez debido a gran número de reacciones adversas.

Productos sintéticos: Se definen también como implantes aloplásticos, e incluyen:

1. Silicona sólida: No parece ser un buen injerto. Nunca lo hemos considerado una opción en nuestro centro, pero nos llama la atención la gran aceptación en Asia donde existe gran experiencia en su uso. Resulta especialmente útil con piel gruesa la que protege y suaviza el efecto de este implante. Sin embargo al ser un material no poroso presenta mayor generación de cápsula cicatricial y mayor riesgo de infección, motivos por los que no ha sido ampliamente implementado en el mundo occidental, con piel algo más delgada ${ }^{7-8}$.

2. Gore-Tex: Ha ocupado un lugar como el material aloplástico de elección, debido a su conformación porosa pero con mínima infiltración y crecimiento de tejido a su alrededor, evitando también la generación de cápsula. Su tasa de infección es menor a 3,2\% y su extrusión menor al $1 \%{ }^{9}$. Esta estadística se aumenta 4 veces en rinoplastía secundaria, reafirmando el concepto de evitar aloplásticos en rinoplastía de revisión.

3. Polietileno poroso de alta densidad: (HDPPE, Medpor) son más rígidos que Gore-Tex, siendo aloplásticos de elección para injertos estructurales, aunque presentan mayor riesgo de extrusión ${ }^{10}$.

4. Reabsorbibles sintéticos: (Lactosorb) Aun en investigación sobre resultado estético y funcional al largo plazo, consiste en un material que se reabsorbe completamente en 1 año, siendo reemplazado por tejido fibroso ${ }^{1}$.

Productos de ingeniería biomédica de tejidos: Continuamente se están probando nuevos tipos de materiales diseñados a nivel molecular para lograr las características ideales, especialmente en la ausencia de respuesta a cuerpo extraño y biointegración. Probablemente el diseño específico a través de ingeniería biomédica de tejidos represente el verdadero futuro en injertos ${ }^{1}$.

El uso de cualquiera de estos injertos se enmarca como un aspecto central dentro una tendencia internacional en rinoplastía a cada vez "hacer menos" y a no "sobre operar", resecando menos tejidos y preservando más estructuras, independiente del abordaje utilizado, sea éste cerrado (intercartilaginosa, transcartilaginosa 0 delivery) 0 abierto.

En el siguiente trabajo presentamos una breve revisión y una muestra de la experiencia en el uso de injertos en rinoseptoplastía de nuestro Comité de Nariz, instancia docente-asistencial existente desde 1998, donde durante un período de 4 meses cada residente en su último año de formación cuenta con la oportunidad de realizar rinoseptoplastías, tutoreado por un otorrinolaringólogo con experiencia en este tipo de procedimiento.

\section{MATERIAL Y MÉTODO}

Estudio descriptivo, retrospectivo realizado entre marzo de 2008 y agosto de 2009 en el Hospital Clínico de la Pontificia Universidad Católica de Chile. Se realizó una revisión sistemática de los protocolos operatorios de la totalidad de los pacientes operados de rinoseptoplastía dentro del Comité de Nariz de dicho centro, bajo el diagnóstico de rinoseptodesviación.

Se evaluaron dentro del protocolo datos epidemiológicos, técnicas e injertos ocupados. Se realizó análisis de frecuencia con SPSS 17.0, separando por tipo de abordaje.

Para evitar redundancia del artículo y para facilitar su lectura, se presenta en la sección de resultados una breve síntesis de las características y técnicas para cada injerto junto a nuestros resultados propiamente tales. 
Tabla 2. Resumen del uso de Injertos en el Comité de Nariz PUC

\begin{tabular}{|c|c|c|c|c|}
\hline & & $\begin{array}{l}\text { Rinoplastía } \\
\text { abierta }\end{array}$ & $\begin{array}{c}\text { Rinoplastía } \\
\text { cerrada }\end{array}$ & $\begin{array}{c}\text { Total } \\
\text { rinoplastías }\end{array}$ \\
\hline & Total casos & $82\left(84 \%{ }^{*}\right)$ & $16\left(16 \%{ }^{*}\right)$ & $98\left(100 \%{ }^{*}\right)$ \\
\hline Injertos estructurales punta nasal & $\begin{array}{l}\text { Vástago } \\
\text { Alar Batten }\end{array}$ & $\begin{array}{c}81\left(99 \%{ }^{* *}\right) \\
3\left(4 \%^{* *}\right)\end{array}$ & $\begin{array}{l}1\left(6 \%{ }^{* *}\right) \\
-\end{array}$ & $\begin{array}{c}82\left(84 \%^{*}\right) \\
\left.33 \%^{*}\right)\end{array}$ \\
\hline Injertos Refinamiento punta nasal & $\begin{array}{l}\text { Escudo } \\
\text { Paragua } \\
\text { Alar rim }\end{array}$ & $\begin{array}{c}27\left(33 \%^{* *}\right) \\
1\left(1 \%^{* *}\right) \\
3\left(1 \%^{* *}\right)\end{array}$ & $\begin{array}{l}- \\
- \\
-\end{array}$ & $\begin{array}{c}27\left(28 \%{ }^{*}\right) \\
1\left(1 \%{ }^{*}\right) \\
1\left(1 \%{ }^{*}\right)\end{array}$ \\
\hline Injertos tercio medio & Espaciador & $17\left(21 \%{ }^{* *}\right)$ & $1\left(6 \%{ }^{\star \star \star}\right)$ & $18\left(18 \%^{*}\right)$ \\
\hline Injerto de dorso & Injerto de nasion & $3\left(4 \%{ }^{* *}\right)$ & - & $3\left(3 \%^{*}\right)$ \\
\hline Tutores tabique & Tutor borde caudal & $3\left(4 \%{ }^{* *}\right)$ & $2\left(13 \%{ }^{* * *}\right)$ & $5\left(5 \%{ }^{*}\right)$ \\
\hline
\end{tabular}

"Porcentaje de todas las rinoplastías. "Porcentajes de las rinoplastías abiertas. " Porcentaje de las rinoplastías cerradas.

\section{RESULTADOS}

Se revisaron los protocolos quirúrgicos de un total de 98 pacientes operados en el período, donde $62 \%$ fueron mujeres (edad entre 16 y 71 años, promedio de 36 años) y $38 \%$ hombres (17 a 46 años, promedio de 27 años).

Con respecto a la vía de abordaje el $84 \%$ de los paciente fue abordado por técnica abierta y el $16 \%$ restante por vía cerrada transcartilaginosa. La totalidad de los injertos utilizados en esta experiencia fueron autólogos en base a cartílago septal.

Para fijar los injertos en todos los casos usamos PDS 5-0 excepto en el injerto de nasion en donde se usó catgut transcutáneo.

En nuestra casuística, al momento de la revisión no hubo complicaciones que se puedan atribuir específicamente al uso de injertos.

\section{Injertos estructurales de la punta nasal}

Vástago: 0 "columelar strut", descrito por Fomon y Farrior, popularizado en 1969 por Anderson ${ }^{11,12}$. Fue utilizado en el $84 \%$ de todas las rinoplastías, de las cuales casi la totalidad fueron rinoplastías abiertas (sólo se colocó un vástago en una rinoplastía cerrada). Consiste en la instalación de un injerto de forma rectangular alargada, en forma de poste entre las cruras mediales de ambos cartílagos alares inferiores (Figura 1). El vástago proyecta y rota la punta, dándole gran soporte estructural y es usado habitualmente en un abordaje abierto, el cual por sí mismo debilita la estructura nasal provocando una caída de la punta si no es corregida. Por este motivo, el vástago es usado de regla en abordaje abierto en nuestro comité. Sus

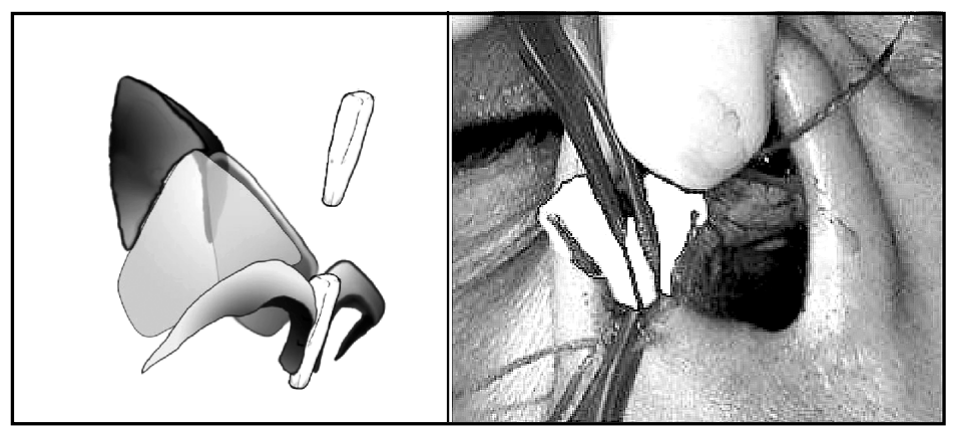

Figura 1. Injerto estructural punta nasal: Vástago. Esquema del injerto entre las cruras mediales de ambos alares inferiores (izquierda) y su forma de colocación (derecha). 
complicaciones son raras e incluyen un ensanchamiento de la columela y la protrusión del injerto, las que no han sido observadas en nuestra casuística hasta el momento.

Refuerzo alar: Su principal función es ser un cartílago de soporte o refuerzo para resolver el colapso alar y la obstrucción valvular que de ello se genera. Consiste en la instalación de cartílagos bilaterales sobre las cruras laterales de los alares inferiores como refuerzo estructural a un alar colapsado, intentando restaurar una posición ideal (Figura 2). En la experiencia de Toriumi en 5 años de uso, resolvió el $98 \%$ de las obstrucciones nasales secundarias a colapso alar ${ }^{12}$. En nuestra casuística, se utilizó en 3 casos (4\%), todos mediante abordaje abierto. Su principal complicación es una sobreocupación sobrealar de tejido fibroso que en la experiencia de Toriumi se resuelve en todos los casos al cabo de 1 año.

\section{Injertos de refinamiento de la punta nasal}

Escudo: "shieldgraft". Descrito por primera vez por Sheen, tiene forma trapezoidal y se posiciona superficial a las cruras mediales en línea media ${ }^{13}$ (Figura 3). Su principal función es lograr una mayor definición de la punta nasal, agudizando sus ángulos (siempre dependiente de la piel del paciente), asegurando simetría de la punta y proyectándola. Sólo se implementó en rinoplastías abiertas, siendo utilizada en el $33 \%$ de ellas ( $y$ en el $28 \%$ de toda la muestra). La complicación más frecuente del escudo es la deformidad estética provocada por la protrusión del injerto. Esto se puede presentar con mayor frecuencia en pieles finas (que por el origen étnico de nuestros pacientes, es menos frecuente de encontrar en nuestra casuística). Al momento de la revisión no se presentaron complicaciones por el uso de este injerto.

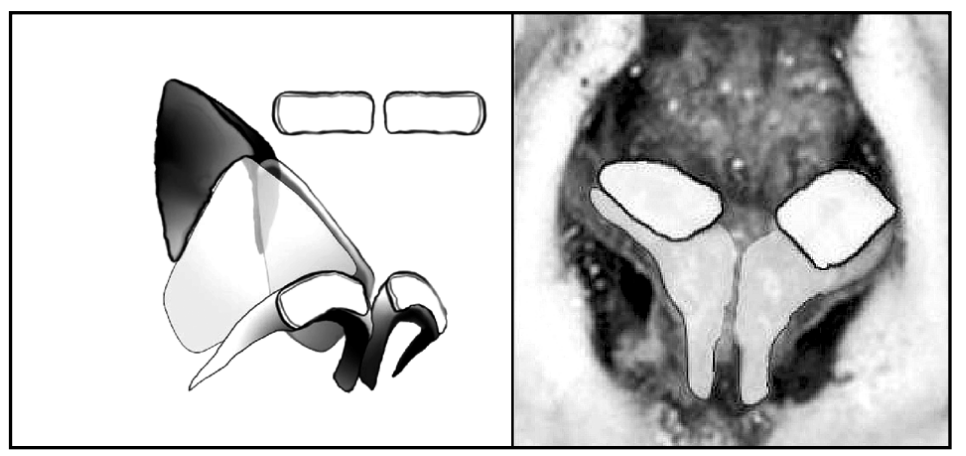

Figura 2. Injerto estructural punta nasal: Alar Batten. Esquema del injerto bilateral (izquierda) posicionado supra-alar, sobre la región más externa de los domos y su prolongación hacia las cruras laterales. Una vez instalados (derecha) corrigen el colapso dado por alares inferiores debilitados.

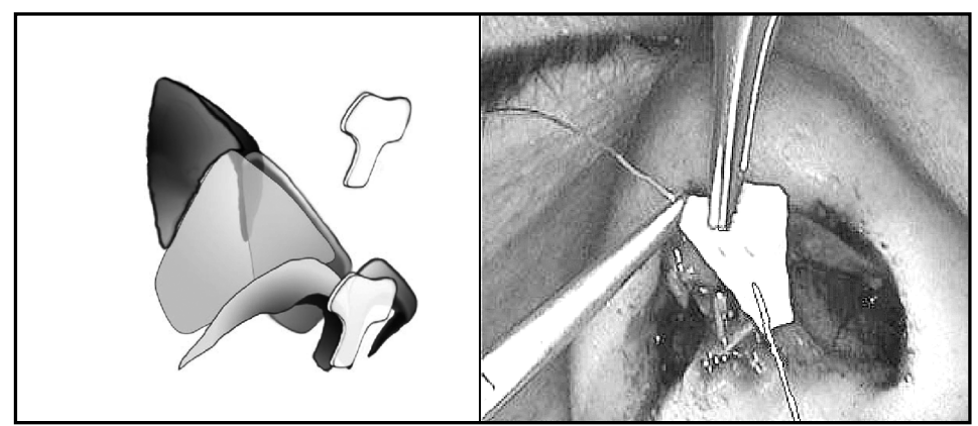

Figura 3. Injerto de refinamiento de punta nasal: Escudo. Esquema del escudo caudal a las cruras mediales de los cartílagos alares inferiores (izquierda) y su forma de colocación (derecha), donde destaca el uso de aguja de carpule para fijar posición del escudo antes de realizar puntos. 
Paraguas: Descrito por Peck, consiste en la inserción de un único cartílago de forma levemente cuadrangular redondeada sobre los domos (Figura 4). Su función es lograr una mayor definición de la punta nasal. Fue usada en un único caso en nuestra casuística en rinoplastía abierta, por no disponer de suficiente cantidad de cartílago septal para hacer un escudo.

Alar rim: Consiste en la inserción de una fina tira de cartílago por la cara interna del borde libre del ala nasal (Figura 5). Intenta corregir una retracción alar leve así como mejorar el contorno y forma alar. Se utilizó en 1 caso (1\%), y fue a través de abordaje abierto.

\section{Injerto tercio medio}

Espaciador: Diseñado por Gunther y Rohrich su intención inicial fue mejorar el pinzamiento del tercio medio. Consiste en colocar un injerto rectangular, entre el lateral superior y el tabique (Figura 6). Sus efectos aparte de mejorar el pinzamiento de tercio medio al usarlo bilateralmente, son mejorar el ángulo valvular, corregir una laterorrinia al usarlo unilateralmente $\mathrm{y} / 0$ corregir una septodesviación del dorso. Fue utilizado en 17 casos en rinoplastía abierta ( $21 \%$ de ellas) y en un caso por abordaje cerrado. En 15 ocasiones fue unilateral, de las cuales en 2 casos se usaron dos injertos uno sobre otro unilateralmente para un mayor efecto. Sólo en un caso su uso fue bilateral. Sus complicaciones son muy raras, e incluyen un ensanchamiento exagerado del dorso y una obstrucción nasal por alteración de la válvula con tejido cicatricial. Ninguna de estas situaciones se vio en nuestra casuística.

\section{Injerto de dorso}

Injerto de nasion: Consiste en un injerto rectangular que tiene por objetivo levantar la zona del dorso a nivel del nasion (Figura 7), la necesidad de utilizar este injerto debe ser evaluado tomando en consideración la altura del dorso que se dejara en relación a la proyección de la punta ("balance

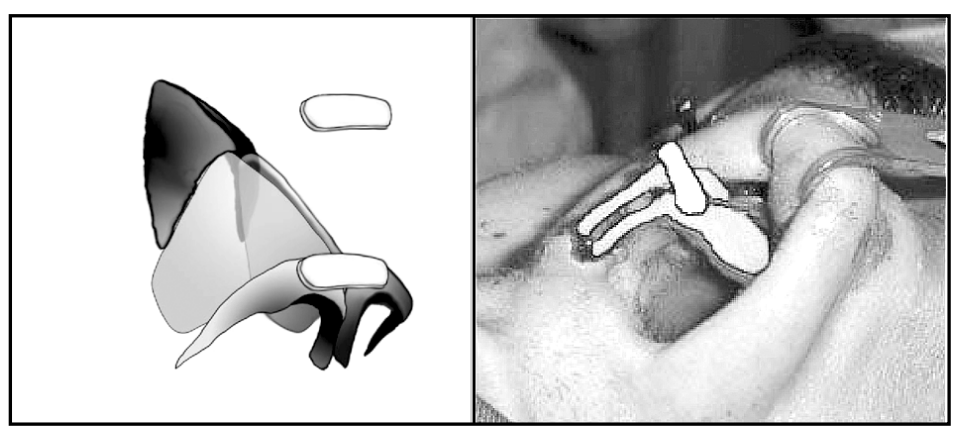

Figura 4. Injerto de refinamiento de punta nasal: Paraguas. Esquema de su posición (izquierda) y una foto de su uso final (derecha).

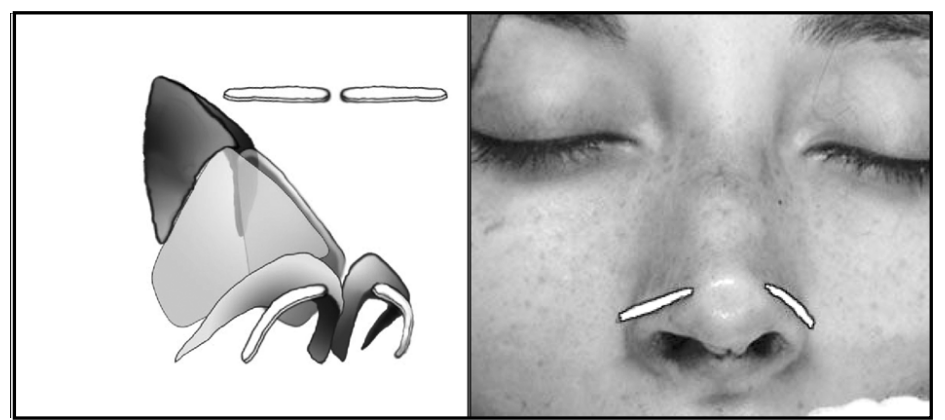

Figura 5. Injerto de refinamiento de punta nasal: Alar Rim. Se observa un esquema de uso de estos injertos rectos para apoyar la forma del ala nasal (izquierda) y una foto con su sobreposición deseada en un paciente (derecha). 


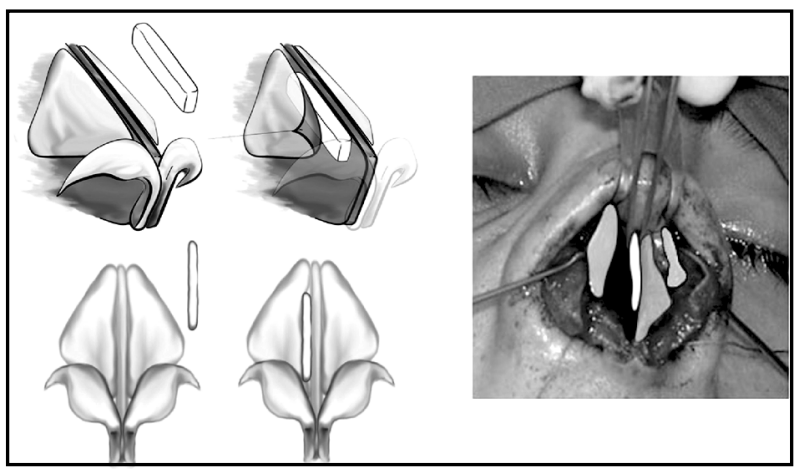

Figura 6. Injerto de tercio medio: Espaciador. Esquema del injerto entre la porción caudal del tabique nasal y el cartílago alar superior (izquierda) y una foto con la forma de inserción (derecha, se nota injerto en blanco, tabique junto a él en gris más oscuro, y laterales superiores en gris algo más claro hacia los lados).

dorso-punta nasal"). Fue usado en 3 casos, todos por abordaje abierto. Este es el único injerto el cual para fijarlo no se usó PDS 5-0, en su lugar usamos catgut 4-0, dado que este punto es sólo transitorio, queda expuesto sobre el dorso nasal (ver Figura 7), y se retira a la semana de la realización del procedimiento.

\section{Injerto en tabique}

Tutor de borde caudal: La mayoría de las septodesviaciones se resuelven resecando las porciones de tabique desviadas, cuidando con mantener un marco de tabique céfalo caudal que mantenga el soporte estructural de la nariz (Figura 8). Cuando la desviación es a nivel del marco caudal, la inserción de un cartílago adosado a él puede corregir o compensar esta desviación. También ayuda a evitar sobrerrotación de la punta, al prolongar el borde caudal del cartílago septal.
Fue usado en 5 casos ( $5 \%$ de todos los casos), 3 en cirugía abierta y 2 en cerrada.

\section{DISCUSIÓN}

La experiencia presentada representa las tendencias del Comité de Nariz de nuestro centro, donde el abordaje abierto es de elección en la gran mayoría de los casos (84\%), salvo que se necesiten correcciones mínimas. En términos generales el abordaje cerrado se prefirió en aquellos pacientes en los cuales no era necesario intervenir la punta nasal, como ocurre por ejemplo en sólo rebaje de la jiba.

En la gran mayoría de los casos (87\%) se utilizó algún tipo de injerto.

En este sentido el uso de injertos sigue las pautas internacionales en donde cada vez se reseca

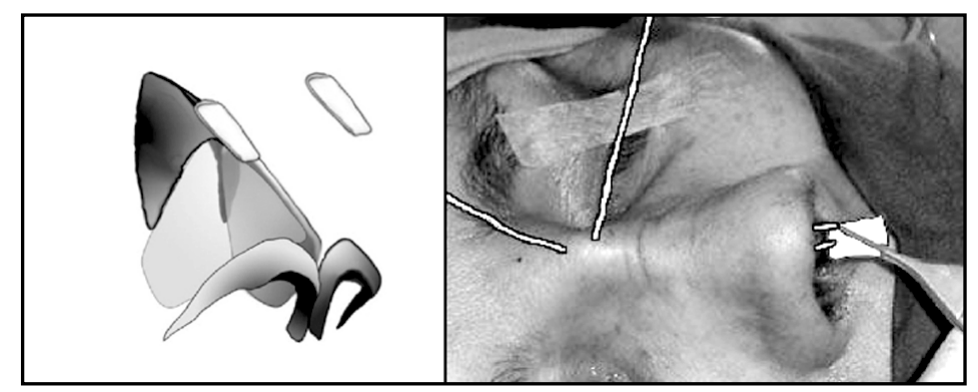

Figura 7. Injerto de dorso: nasion. Esquema del injerto (izquierda) y forma de colocación (derecha), donde destaca el uso de puntos guías en piel en lugar escogido, usados para traccionar injerto. Estos puntos serían retirados en el posoperatorio. 


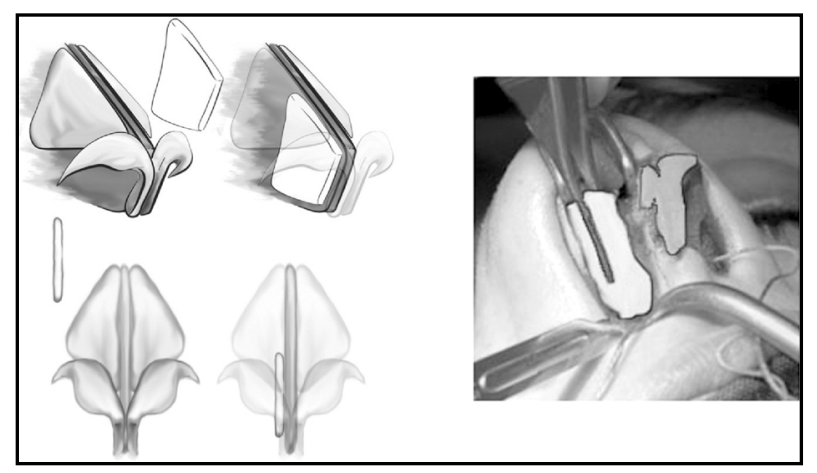

Figura 8. Injerto de tabique: tutor de punta nasal. Esquema de uso, adosado a la punta del tabique (izquierda) y foto de forma de inserción, desplazando alares en el procedimiento (derecha).

menos, y enfocados a "rellenar" para lograr los resultados estéticos perseguidos.

El material de elección fue injerto autólogo de tabique septal en todos los casos, por su facilidad para ser tallado, su inocuidad biológica, rigidez, durabilidad y maleabilidad. Con esto se evitó usar otros campos quirúrgicos como pabellón auricular o parrilla costal.

El vástago fue ampliamente usado, no sólo para dar un soporte y estabilidad estructural, sino también para lograr un manejo controlado de la proyección y rotación de la punta nasal. Lo consideramos un injerto que debe ir de regla en una rinoplastía abierta, salvo que otra estructura como el borde caudal tome la función de compensar el debilitamiento de la punta propia de este tipo de abordaje. Creemos que la estabilidad en el largo plazo de la punta nasal depende fundamentalmente de este injerto.

En segundo lugar de frecuencia se usó el escudo. Este es de gran ayuda para lograr una mayor definición de la punta, especialmente frente a puntas con ángulos poco claros y más "redondeadas" de lo deseado. La definición perseguida se asocia a la imagen de "doble quiebre" de los domos, buscando un aspecto armonioso. Este tipo de injerto es recomendable sólo en personas con piel gruesa.

Si consideramos que nuestra población de origen principalmente hispanoamericano presenta pieles gruesas y cartílagos alares débiles, este injerto representa un aporte valioso a la definición y estructura de la punta, con una piel que permite su uso.
En el mismo sentido, se debe tener especial precaución en personas de origen caucásico los que tiene en su mayoría piel fina.En estos casos, el injerto se podría protruir marcándose y deformando la nariz.

El injerto espaciador es bastante útil cumpliendo funciones tanto estéticas como funcionales, donde mejora el ángulo valvular, zona de mayor resistencia al flujo de aire. La tendencia en el comité durante el período revisado, fue a usarlo cada vez más. Se debe tener cuidado ya que se describen dentro de sus complicaciones, el compromiso del ángulo valvular por retracción cicatricial. Por otro lado, permite prevenir el nunca deseado pinzamiento del tercio medio o nariz en "v" invertida. Este injerto debería ser usado profilácticamente cuando existen huesos propios cortos.

Al ser usado en conjunto con osteotomías de la pirámide nasal, representa un importante apoyo al corregir laterorrinias, lo que a nuestro juicio es uno de los mayores desafíos en rinoplastía.

El resto de los injertos descritos fueron usados en casos puntuales con necesidades específicas. En todos estos casos la implementación de estos injertos logró resolver de forma satisfactoria el problema.

Dentro de la opinión del Comité de Nariz de nuestro centro, y basándose en la experiencia descrita, el cirujano de rinoseptoplastía debe tener un conocimiento profundo y sólido en el uso de injertos en esta cirugía, con el fin de lograr mejores y reproducibles resultados estéticos y funcionales. 


\section{BIBLIOGRAFÍA}

1. Ansari $\mathrm{K}$, Asaria J, Hilger $P$ y cols. Grafts and implants in rhinoplasty - Techniques and longterm results. Op Tech Otolaryngol Head Neck Surg 2008; 19: 42-59.

2. EPPRECHT R. Rinoplastia Abierta. Editorial Cantabria 1999. Capitulo XIII: Injertos e Implantes; 152-7.

3. Constantino PD, Freedman CD. Soft tissue augmentation and replacement in the head and neck: General considerations. Otolaryngol Clin N Am 1994; 27: 1-12.

4. Tardy ME, Denny J, Fritsch MH. The versatile cartilage autograft in reconstruction of the nose and face. Laryngoscope 1985; 95: 523-33.

5. PORTER JP. Grafts in rhinoplasty, alloplastic vs autogenous. Arch Otolaryngol Head Neck Surg 2000; 126: 558-61.

6. BuRke AJ, Wang TD, Cook TA. Irradiated cartilage grafts in the nose. A preliminary report. Arch Otolaryngol Head Neck Surg 2004; 119: 24-30.
7. Shirakabe Y, Suzuki Y, Lam SM. A systematic approach to rhinoplasty of the Japanese nose. A thrirty-year experience. Aesthetic Plast Surg 2003; 27: 221-31.

8. Tham C, Lal YL, Weng CJ et al. Silicone augmentation rhinolasty in an Oriental population. Ann Plast Surg 2005; 54: 1-5.

9. Scoenrock LD, RepucCl AD. Correction of subcutaneous facial defects using Gore-Tex. Fac Plast Surg Clin N Am 1994; 2: 373-88.

10. Romo T, Sclafani A, SabinI AP y cols. Nasal reconstruction usng porous polyethilene implants. Facial Plast Surg 2000; 16: 55-61.

11. Anderson J. Personal Techniques of Rinoplasty. Otolaryngol Clin N Am 1975; 8: 599.

12. TORIUMI DM Y coLs. Use of alar batten graft for correction of nasal valve collapse. Arch Otolaryngol Head Neck Surg 1997; 123: 802-8.

13. Moore E. Rhinoplasty, Tip Ptosis: Treatment. Medicine Specialties. Actualizado 2 de Septiembre 2009.

Dirección: Dr. Santiago Cornejo Sch. Hospital Naval Viña del Mar Subida Alessandri S/N, Viña del Mar E mail: chagocornejo@yahoo.com 